\title{
Getting From $A$ to IRB: Developing an Institutional Review Board at a Historically Black University
}

\author{
Daniel L. Howard, \\ Shaw University \\ Carlton L. Boyd, \\ Shaw University \\ Daniel K. Nelson, and \\ University of North Carolina at Chapel Hill \\ Paul Godley \\ University of North Carolina at Chapel Hill
}

\begin{abstract}
Shaw University, the oldest historically black college or university in the southern USA, recently partnered with the University of North Carolina at Chapel Hill, a major research institution in North Carolina, to further develop Shaw's research infrastructure. One aim of the partnership involved establishing a human research ethics committee and an accompanying administrative structure and research ethics education program. This paper describes the process of developing an entire human research protection program de novo through collaboration with and mentoring by the members of the human research protection program at a nearby major research institution. This paper provides a detailed description of the aims, procedures, accomplishments, and challenges involved in such a project, which may serve as a useful model for other primarily teaching institutions wishing to develop research infrastructure and ethical capacity.
\end{abstract}

\section{Keywords}

historically black colleges and universities; research infrastructure; partnering; research ethics committee; REC; Institutional Review Board; IRB

Historically black colleges and Universities (HBCUs) have traditionally been known for their supportive educational and teaching experiences. Many HBCUs have focused little on research in deference to their primary educational missions (Carey et al., 2005). Faculties at HBCUs typically have large teaching loads and little time for research. There are 105 HBCUs in the United States (U.S. Department of Education [DOE], 2005), and in 2009, only 17 of the private HBCUs had Institutional Review Boards ${ }^{1}$ (IRBs) registered with the U.S. Department of Health and Human Services (DHHS) Office for Human Research Protections (OHRP). OHRP provides guidance, education, and compliance oversight to IRBs that oversee research conducted or supported by the DHHS (U.S. DHHS, 2005).

\footnotetext{
(C) 2010 BY JOAN SIEBER. ALL RIGHTS RESERVED.

Correspondence should be addressed to Daniel L. Howard, 118 East South Street, Raleigh, NC 27601. Phone: 919-755-7292; danielhoward@ shawu.edu.

${ }^{1}$ The terms Human Research Ethics Board (HREB), Research Ethics Board (REB), and Institutional Review Board (IRB) are used interchangeably in this article.
} 
Shaw University (Shaw), the oldest historically black college in the southern USA, was founded in 1865 and is a private, coeducational, liberal arts institution. It currently enrolls approximately 2,800 undergraduate and graduate students. Since 2000, Shaw has moved from a campus with a limited number of external grant awards by a few investigators who had little assistance with grant preparation to a fully staffed Office of Research and Sponsored Programs (ORSP) with grant funds totaling over \$20 million in 2006. The increase in funded awards over a relatively short period of time prompted Shaw to review the need for establishing a mechanism on campus to review and approve research involving human subjects.

Shaw opted to form an IRB to review not only federally sponsored research, but all research conducted on campus. The goal was supported by a grant obtained through a partnership formed by researchers at Shaw and the University of North Carolina at Chapel Hill (UNC$\mathrm{CH})$. A Center of Excellence in Partnerships for Community Outreach, Research on Health Disparities and Training (Project EXPORT) was funded in 2002 by the National Institutes of Health (NIH) National Center on Minority Health and Health Disparities (NCMHD). The aims of the Project EXPORT grant were multifaceted and included developing a partnership that would rely on the extensive research experience of UNC-CH faculty to mentor and develop a new pool of health disparities researchers at Shaw. A research infrastructure core was established through the Project EXPORT grant at Shaw, and was given the tasks of enhancing the university's existing research infrastructure by upgrading its grants management office and creating an independently functioning IRB by drawing on a comprehensive training plan by our UNC-CH partners. The following description of the activities involved in developing the IRB may benefit other institutions of higher learning that wish to develop IRBs of their own.

\section{Establishing the Partnership}

For Shaw, establishing an IRB required the guidance of well-trained and experienced personnel working in the field of human research ethics. The partnership with UNC-CH provided the opportunity. Since 2000, Shaw and UNC-CH have collaborated on multiple small contracts, research projects, and academic endeavors that helped to establish a sense of trust and mutual understanding between the two institutions (Carey et al., 2005). With the added advantage of geographic proximity (approximately 30 miles), Shaw's investigators and staff could interact face-to-face as needed with researchers from UNC-CH.

There were multiple meetings between the Shaw IRB development staff and the Director of the UNC-CH Office of Human Research Ethics (OHRE) early in the development process. Early discussions concerned government regulations of human research, other documents relevant to human research ethics, and information relevant to establishing an IRB. The Director of UNC-CH OHRE was available for questions on a continuous basis.

The President of Shaw entered into a Federalwide Assurance (FWA) for the Protection of Human Subjects for Domestic (U.S.) Institutions. This assurance indicates that all research at Shaw will be held to U.S. federal standards for the protection of human subjects. Next, Shaw and UNC-CH executed an IRB Authorization Agreement, i.e., an agreement that allows one institution to rely on another institution for review, approval, and oversight of research. In this case, UNC-CH agreed to provide review of all Shaw's research projects until Shaw was able to establish its own IRB. Shaw's investigators were given the opportunity to submit IRB applications to any of the eight UNC-CH IRBs. The agreement also laid out a plan for transition after Shaw's IRB members were well-trained and prepared to review research independently. 
According to a June 2000 requirement issued by NIH, all investigators submitting NIH grant applications or receiving awards for research involving human subjects must receive education on the protection of human research participants (National Institutes of Health, 2000). Many institutions, including UNC-CH, broadened these requirements to all human subjects, regardless of funding. To meet its initial training needs, UNC-CH relied on the Collaborative IRB Training Initiative (CITI) hosted online by the University of Miami (https://www.citiprogram.org/Default.asp?). This web-based course includes a series of comprehensive modules on the protection of human research subjects. Shaw's investigators were allowed access to CITI through the partnership established with UNC-CH.

Shaw's preparation also included regular meetings with UNC-CH staff to observe their entire review and administrative procedures. Shaw's staff attended UNC-CH administrative meetings as well as several IRB committee meetings at UNC-CH to observe experienced IRBs in practice. Also valuable was the interaction with IRB administrators and chairpersons to discuss specific IRB applications by Shaw investigators.

\section{Forming the Committee}

One staff member at Shaw was selected as the interim IRB administrator to manage the dayto-day operation of the OHSP, ensure that all Shaw faculty and staff involved in research completed the human subjects protection training certification, present IRB procedural workshops for Shaw employees, and recruit IRB committee members. There were many challenges inherent in the task of building this infrastructure from the ground up. The IRB administrator introduced the development of the IRB at a Shaw campus-wide faculty meeting at the start of the fall 2003 semester. Subsequent to the meeting, three e-mails were sent to all faculty and staff over a one-month period describing the IRB, the time commitment necessary to participate, and the role of the IRB committee members. The most challenging facet in constructing the IRB committee was recruiting IRB members because of their heavy teaching load; release time had to be approved by each member's department chairperson so their schedules would permit for them to participate in meetings without conflicting with their classes. Interested individuals agreed to attend an informational session. It soon became apparent that the IRB needed to have the status of an "official" campus committee (a committee approved by the Executive Vice President of Shaw) so that serving on the IRB would fulfill the faculty's contract requirement of serving on at least one such committee. The timeline for implementation of a fully functioning IRB started September 2003 and ended November 2005.

Nine IRB members representing a range of backgrounds, expertise, and cultural sensitivities were recruited from Shaw's faculty and staff to satisfy regulatory requirements regarding varying backgrounds to support complete and adequate review of research activities commonly conducted at Shaw. The initial members of the committee ranged from professors in Sociology, English, Accounting, and Business to the Assistant Dean of the graduate education program. In addition to these University employees, an unaffiliated committee member was recruited, a recently retired assistant principal from a local high school.

Recruiting an IRB Chair was a challenge as well. The Chair needed to be a respected member of the campus community who had significant leadership qualities to guide and defend the decisions made by the IRB. Between the time when Shaw's IRB became an independent entity in 2005 and the identification of the IRB Chair, the IRB Administrator served as interim chair and passed the administrative duties to another IRB staff member. In order to attract a strong person to the position, $25 \%$ release time from other academic duties was requested for that individual. Despite this selling point, none of the initial IRB members accepted the offer; consequently, OHSP staff contacted Shaw's Faculty Development Coordinator for assistance in identifying a Chair. The Coordinator identified a faculty 
member who had prior experience on an IRB and who was enthusiastic about the position. The faculty member met with OHSP staff on several occasions, observed some IRB meetings, and assumed the role of Chair in the fall of 2006.

\section{Training the Committee}

The Shaw University IRB Administrator and committee members needed to be educated in the ethical principles, regulations, and guidelines that govern human subjects research. These educational materials included international documents (the Nuremburg Code and the Declaration of Helsinki), national (U.S.) documents (Belmont Report, 45 Code of Federal Regulations 46), and educational and reference materials for IRB decision-making and management. The DHHS OHRP and the UNC-CH OHRE websites were reviewed. Based on these printed materials and websites, policies and procedures to guide the day-to-day activities of the Shaw IRB were written. In addition, the IRB Administrator attended the national conference sponsored by Public Responsibility in Medicine and Research (PRIM\&R), a national organization committed to advancing ethical standards in research.

The Shaw IRB committee members continued their training by attending a summer course on Responsible Conduct of Research in 2004 sponsored by the General Clinical Research Center at UNC-CH. Shaw IRB members rotated through the IRBs at UNC-CH to observe convened meetings of active experienced committees. Some also attended IRB meetings at another nearby major institution. They attended events hosted by the North Carolina Consortium for Protection of Human Subjects, which was developed from an NIH program enhancement grant to UNC-CH. In 2005, some of the Shaw IRB staff and committee members attended two of the Consortium's Regional Conferences held at two HBCUs across the state of North Carolina.

As an additional means of continuing education, articles from the journal IRB: Ethics and Human Research were distributed to IRB members at regular IRB meetings.

\section{Administrative Structure}

At the outset of Project EXPORT, it was recognized that a stand-alone IRB committee would not function efficiently without a supporting office to provide information, field questions, document IRB meeting minutes, distribute letters from the IRB to investigators, handle the intake and processing of applications, and other organizational matters. Since such an office did not exist at Shaw, Project EXPORT developed a document at Shaw titled, "Establishment of the Office of Human Subjects Protection (OHSP)," and disseminated it to Shaw's administration, outlining the importance of the Federalwide Assurance, the IRB Authorization Agreement between UNC-CH and Shaw, educating the Shaw community on the necessity of protecting human research subjects, and the process for submitting proposals for IRB review.

In 2004, the Shaw administration moved to approve its Office of Human Subjects Protection (OHSP). A temporary plan needed to be developed for investigators who required IRB reviews while IRB members were training and before Shaw's IRB became an independent entity. A brochure was created, "HBCU IRB Submission Instructions for Investigators," which described the interim process for submitting IRB applications to UNC-CH's IRBs through the Shaw OHSP and was distributed to all faculty members. Shaw's Office of Research and Sponsored Programs (ORSP) was utilized to further inform investigators of the new processes and to inform the OHSP when new grant awards had been issued that would have human subject involvement.

Prior to initiating review of research from Shaw's investigators, the OHSP developed standard operating procedures (SOPs) essential to the effective management of the IRB, 
modeled after parallel procedures at UNC-CH. A Research Investigator's Guide to the IRB, an OHSP Administrative Procedures manual, and a website (http://www.shawu.edu/IRB/new/Review\%20Process.html) were developed containing all the essential information for investigators. The goal was to make the OHSP and IRB two entities that everyone on campus_-faculty, staff, and students—could access with ease.

Efforts were made to identify all protocols and IRB applications from previous and current studies to get an accounting of activity on campus that required IRB review. OHSP staff also wanted to ensure that all investigators on campus and their key personnel were trained in human research protections. One of the first databases developed in the OHSP tracked the names of individuals and the type, name, and date of the training they received. OHSP developed an additional database to track and store all the information. The purpose was to build a history of each application and the resulting research. In addition, the OHSP had the task of developing job descriptions for key players, such as the IRB chairperson, IRB administrator, and IRB committee member. Other essential tasks in the new office included creating files for each application, and developing forms required for submission of an IRB application.

According to Gunsalus (1993), "The single most influential component in an institutional culture of research integrity is institutional leadership committed to ethical conduct." She also asserts that the most effective plan is to create policy that fits the institution in question. This institutional support or leadership should be apparent by its commitment to (1) creating an ethics infrastructure, (2) assuring adequate resources, (3) supporting IRB members, and (4) educating all involved (Sugarman, 2000). Shaw's commitment to research integrity was expressed in a number of ways, including the following: On March 24, 2004, the president of Shaw formally announced that the University would accept the responsibility of assuring that all activities related to federally and non-federally supported human subject research would comply with the terms of the Federalwide Assurance (FWA) for the Protection of Human Subjects for Domestic (U.S.) Institutions. In addition, the UNC-CH Office of Human Research Ethics and the Co-Director for the EXPORT Partnership IRB section and his staff provided ongoing consultation for IRB development. The Center Director, CoDirector, and Core Directors were responsible for scientific leadership, effective communication, making the IRB visible to Shaw faculty and students, monitoring partnership outcomes, ensuring oversight in ethical conduct, monitoring all budgetary matters, and maintaining records and reports. The leadership team met monthly to discuss progress and steps to move forward with the development of the IRB. Support for the IRB was displayed by the Shaw administration's provision of release time for the chairperson and faculty credit for participating in a campus committee as IRB members. The OHSP also consulted with the university attorney about liability insurance for all IRB members, and the IRB was added to a Shaw insurance policy. It was also important to establish this contact with Shaw's counsel in the event that legal issues arose in the future.

Shaw's commitment to educate the campus community in the ethics of research has been displayed in numerous ways. Early in the process, OHSP staff attended a university-wide faculty meeting and presented information pertaining to the IRB and the new procedures on campus; they distributed a letter explaining the new direction, brochures with instructions for submitting IRB applications, and a list of seminars geared toward training. Currently, the OHSP has a mandatory educational requirement for investigators prior to the commencement of any research involving humans. Investigators and key research personnel must complete on-line training in the ethics of research involving human subjects every two years. The OHSP hosted a campus community discussion based on the video, "We All Have Our Reasons: Community Perceptions of HIV Vaccine Research." The video examined the perceptions of government-sponsored research from the perspective of three different 
communities. A discussion followed in which participants, including IRB members, were allowed the opportunity to discuss their opinions of research in general and the varying opinions of people who have participated in research or have heard about research taking place in their communities, as well as the factors involved in making the decision to participate in research.

\section{Challenges and Conclusions}

The Office of Human Subjects Protection and IRB were developed to supplement the Shaw research infrastructure by mentoring and training faculty and staff who would become the IRB committee. Shaw investigators and faculty members have embraced health disparities and minority health research, conducting pilot-research studies in HIV/AIDS, diabetes, cardiovascular disease, and quality-of-care issues in African Americans. UNC-CH mentors have continued to provide scientific leadership and direction to the Shaw IRB as well as the researchers who utilize the IRB for protocol approval. The OHSP and IRB have been a catalyst for heightened interest in expanded research activity at Shaw with more faculty members considering involvement in research activities. In 2005, the Shaw IRB registered with the federal Office for Human Research Protections (OHRP), recognizing its readiness to function independently. The FWA was initially linked to all of the UNC-CH IRBs but is now able to cite Shaw's own IRB. Since Shaw's IRB registration as an independent entity, eighty-six IRB applications have been submitted to the Office of Human Subjects Protection and the amount of funding for health research has greatly increased from $\$ 1.1$ million in 2005 (12 grants submitted) to $\$ 3.9$ million in 2008 (30 grants submitted).

Shaw has a low-volume IRB compared to the IRBs at UNC-CH in terms of the number of IRB protocols submitted and reviewed each year. However, it requires considerable resources to establish and sustain an effective independent IRB, to maintain federal and university compliance, and to protect human subjects. These resources include the following: training and education (conferences, CITI on-line course, newsletters), time commitments of IRB faculty and staff (IRB administrator, IRB Chair, committee members), and supplies (travel, paper, phones, photocopying). It clearly would be more cost-effective to continue the IRB Authorization Agreement with UNC-CH, however, a strict cost-benefit analysis would ignore the intangible benefits of an independent IRB at a minority-serving institution such as Shaw. The benefits include the Shaw community being viewed as having a decisive voice in the research process, the increased involvement in the research process by principal investigators and the IRB committee members, and the opportunity to train students to participate in research. When these intangible benefits are taken into account, it is likely that many teaching institutions seeking to develop research programs would opt to develop their own IRBs.

One of the greatest challenges in the process of developing Shaw University's IRB and human research protection program was the general lack of investigator experience with completing IRB applications and navigating the review process. The University's move toward developing new research investigators coincided with the development of the IRB. Many new investigators were not familiar with the entire process of developing, submitting, and responding to requests from the IRB. This inexperience, coupled with the new investigators' substantial teaching responsibilities and other campus commitments, made it a challenge to prepare adequate IRB applications. Initially, when IRB applications were under review by UNC-CH, Shaw OHSP staff had numerous contacts with research investigators. There were many follow-up e-mails and phone calls to investigators to ensure that forms were filled out satisfactorily. Investigators also received assistance with amendment preparation; review of individual protocols; consent form preparation; submission deadlines; and calls to UNC-CH IRBs to clarify questions. 
Shaw's research infrastructure developed at a fast pace with the Project EXPORT grant serving as the catalyst. The infrastructure now includes an IRB; the Center for Biostatistics and Data Management; the Center for Survey Research; the Institute for Health, Social, and Community Research; a health sciences library; and a research building. Shaw and UNC-CH have collaborated on additional research endeavors, such as the Shaw UNC-CH Center for Prostate Cancer Research where a Shaw faculty member served as the primary investigator. The universities have been awarded a second Project EXPORT grant that focuses on primary research and designates primary roles for Shaw's Center for Biostatistics and Data Management and Center for Survey Research.

\section{Best Practices}

To successfully establish a Human Research Protection Program and an IRB at a university similar to Shaw, an important first step would be to conduct a cost analysis to determine the overall cost of operating an IRB and whether it is financially feasible for the institution to support. These costs may include the following: (1) personnel; (2) training and education; (3) equipment and supplies; and (4) space. If it is estimated that the resources are available to establish and sustain an IRB, the next recommended step would be to partner with an experienced major research institution for guidance and mentorship. An IRB Authorization Agreement with the partnering research institution allows all protocols submitted by the developing university's principal investigators to be reviewed by the mentoring institution until the developing institution's IRB is in operation. A partnership with the research institution also allows the developing university's IRB staff to observe and learn the research institution's procedures, including the administration of its IRB, and provides an opportunity for the members of the developing university's team to observe actual IRB meetings at the research institution. Another important component in the development of an IRB is garnering buy-in from the university president, administration, department chairpersons, faculty, and staff. Educating them about the significance of human research oversight is vital to gaining their cooperation as the IRB moves from the stage of development to functionality. At universities similar to Shaw where there is limited research infrastructure and a lack of investigator experience in completing IRB applications and understanding of the sensitive nature of human subjects protection, there must be an effort to educate investigators.

\section{Research Agenda}

Once an academic institution has developed an IRB, a campus-wide evaluation is appropriate for evaluating the various facets of its operation from the perspective of its staff and users. Receiving feedback from investigators and students about their experiences, satisfactions, and dissatisfactions with the IRB allows the institution's research administration to learn what works and what needs to be improved. Such evaluation could also assess the change in research done by faculty, staff, and students and the change in grant funding.

\section{Educational Implications}

The purpose of this article is to detail the steps taken by Shaw University to develop an effective infrastructure for protecting human subjects of research. The particular educational materials and experiences that enabled Shaw to achieve its goal may differ somewhat from those appropriate to other developing institutions, particularly those in other countries. However, the general educational needs of other developing institutions would be similar and an appropriate parallel set of materials and experiences might be modeled on those described herein. 


\section{Acknowledgments}

The development of the Shaw University Institutional Review Board was supported by the National Center on Minority Health and Health Disparities Grants P60 MD000244 and P60 MD000239.

\section{References}

Carey TS, Howard DL, Goldmon M, Roberson JT, Godley PA, Ammerman A. Developing effective inter-university partnerships and community-based research to address health disparities. Academic Medicine. 2005; 80(11):1039-1145. [PubMed: 16249303]

Gunsalus CK. Institutional structure to ensure research integrity. Academic Medicine. 1993; 68(9):S33-S38. [PubMed: 8373489]

National Institutes of Health. The National Research Act, Title II-Public Law 93-348. 2000. Retrieved October 29, 2009 from http://ohsr.od.nih.gov/guidelines/belmont.html

Shaw University Office of Research and Sponsored Programs. Shaw University Office of Research \& Sponsored Programs funded research report, 2001-2006. Raleigh, NC: Office of Institutional Research; 2006.

Sugarman J. The role of institutional support in protecting human research subjects. Academic Medicine. 2000; 75(7):687-692. [PubMed: 10926018]

U.S. Department of Education, Office of Postsecondary Education, White House Initiative on Historically Black Colleges and Universities. The mission continues: Annual report to the President on the results of participation of historically black colleges and universities in Federal programs, 2002-03. Washington, D.C: President's Board of Advisors on Historically Black Colleges and Universities; 2005.

U.S. Department of Health and Human Services. Code of Federal Regulations, Title 45, Public Welfare, Part 46, Protection of Human Subjects. 2005. Retrieved September 23, 2005 from http://www.hhs.gov/ohrp/humansubjects/guidance/45cfr46.htm

U.S. Department of Health and Human Services, Office for Human Research Protections. OHRP fact sheet. 2005. Retrieved October 6, 2006, from http://www.hhs.gov/ohrp/about/ohrpfactsheet.pdf

U.S. Department of Health and Human Services, Office for Human Research Protections (OHRP). Registration of an Institutional Review Board (IRB) or Independent Ethics Committee (IEC). 2006. Retrieved June 23, 2009 from http://www.hhs.gov/ohrp/assurances/index.html

\section{Biographies}

Daniel L. Howard is Director of the Office of Human Subjects Protection (OHSP) and Director of the Institute for Health, Social, and Community Research (IHSCR) at Shaw University. Dr. Howard's research interests include the examination of epidemiologic patterns of health outcomes that disproportionately affect African Americans, health services research, and health policy.

Carlton L. Boyd is IRB Administrator for the Shaw University Institutional Review Board and Project Assistant for several research studies in the Carolina-Shaw Project EXPORT partnership. His current research focuses on engaging African American churches in the dissemination of health culturally effective health information and assessing these churches' readiness to engage in research.

Daniel K. Nelson is Director for the Office of Human Research Ethics and Associate Professor of Social Medicine and Pediatrics at the University of North Carolina, Chapel Hill.

Paul Godley is Associate Professor of Medicine at the University of North Carolina, Chapel Hill; Director of the Program on Ethnicity, Culture, and Health Outcomes (ECHO); and Director of the Carolina-Shaw Partnership for the Elimination of Health Disparities. His 
research interests focus on a spectrum of population-based investigations of prostate cancer and ethnic and racial health disparities. 